\title{
Existence, Nonexistence, and Stability of Solutions for a Delayed Plate Equation with the Logarithmic Source
}

\author{
Hazal Yüksekkaya, ${ }^{1}$ Erhan Pișkin, ${ }^{1}$ Salah Mahmoud Boulaaras $\mathbb{D}^{2,3}$ \\ Bahri Belkacem Cherif ${ }^{D}{ }^{2,4}$ and Sulima Ahmed Zubair ${ }^{2,5}$ \\ ${ }^{1}$ Department of Mathematics, Dicle University, Diyarbakir, Turkey \\ ${ }^{2}$ Department of Mathematics, College of Sciences and Arts, ArRas, Qassim University, Saudi Arabia \\ ${ }^{3}$ Laboratory of Fundamental and Applied Mathematics of Oran (LMFAO), University of Oran 1, Oran, 31000 Oran, Algeria \\ ${ }^{4}$ Preparatory Institute for Engineering Studies in Sfax, Tunisia \\ ${ }^{5}$ Department of Mathematics, College of Education, Juba University, Sudan
}

Correspondence should be addressed to Sulima Ahmed Zubair; sulimaa2021@gmail.com

Received 12 May 2021; Accepted 16 June 2021; Published 28 June 2021

Academic Editor: Kamyar Hosseini

Copyright (c) 2021 Hazal Yüksekkaya et al. This is an open access article distributed under the Creative Commons Attribution License, which permits unrestricted use, distribution, and reproduction in any medium, provided the original work is properly cited.

In this work, we study a plate equation with time delay in the velocity, frictional damping, and logarithmic source term. Firstly, we obtain the local and global existence of solutions by the logarithmic Sobolev inequality and the Faedo-Galerkin method. Moreover, we prove the stability and nonexistence results by the perturbed energy and potential well methods.

\section{Introduction}

In this article, we consider a plate equation with frictional damping, delay, and logarithmic terms as follows:

$$
\begin{cases}u_{t t}+\Delta^{2} u+\alpha u_{t}(t)+\beta u_{t}(x, t-\tau)=u \ln |u|^{\gamma} & \text { for }(x, t) \in \Omega \times(0, \infty), \\ u(x, t)=\frac{\partial u(x, t)}{\partial v}=0 & \text { for }(x, t) \in \partial \Omega \times(0, \infty), \\ u(x, 0)=u_{0}(x), u_{t}(x, 0)=u_{1}(x) & \text { for } x \in \Omega, \\ u_{t}(x, t)=j_{0}(x, t) & \text { for }(x, t) \in \Omega \times(-\tau, 0),\end{cases}
$$

where $\Omega \subset R^{N}, N \geq 1$, is a bounded domain with smooth boundary $\partial \Omega . \tau>0$ denotes time delay, and $\alpha, \beta$, and $\gamma$ are real numbers that will be specified later. Generally, logarithmic nonlinearity seems to be in supersymmetric field theories and in cosmological inflation. From quantum field theory, that kind of $\left(u|u|^{p-2} \ln |u|^{k}\right)$ logarithmic source term seems to be in nuclear physics, inflation cosmology, geophysics, and optics (see $[1,2])$. Time delays often appear in various problems, such as thermal, economic, biological, chemical, and physical phenomena. Recently, partial differential equations have become an active area with time delay (see $[3,4])$. In 1986, Datko et al. [5] indicated that, in boundary control, a small delay effect is a source of instability. Generally, a small delay can destabilize a system which is uniformly stable [6]. To stabilize hyperbolic systems with time delay, some control terms will be needed (see [7-9] and references therein).

For the literature review, firstly, we begin with the studies of Bialynicki-Birula and Mycielski [10, 11]. The authors investigated the equation with the logarithmic term as follows:

$$
u_{t t}-u_{x x}+u-\varepsilon u \ln |u|^{2}=0
$$

where the authors proved that, in any number of dimensions, wave equations including the logarithmic term have localized, stable, soliton-like solutions.

In 1980, Cazenave and Haraux [12] studied the equation as follows:

$$
u_{t t}-\Delta u=u \ln |u|^{k}
$$


where the authors in [12] proved the existence and uniqueness of the solutions for equation (3). Gorka [2] obtained the global existence results of solutions for one-dimensional equation (3). Bartkowski and Gŏrka [1] considered the weak solutions and obtained the existence results.

In [13], Hiramatsu et al. studied the equation as follows:

$$
u_{t t}-\Delta u+u+u_{t}+\left|u^{2}\right| u=u \ln u
$$

In [14], Han established the global existence of solutions for equation (4).

In [15], Al-Gharabli and Messaoudi were concerned with the plate equation with the logarithmic term as follows:

$$
u_{t t}+\Delta^{2} u+u+h\left(u_{t}\right)=k u \ln |u|
$$

They established the existence results by the Galerkin method and obtained the explicit and decay of solutions utilizing the multiplier method for equation (5).

In [16], Liu introduced the plate equation with the logarithmic term as follows:

$$
u_{t t}+\Delta^{2} u+\left|u_{t}\right|^{m-2} u_{t}=|u|^{p-2} u \log |u|^{k}
$$

The author proved the local existence by the contraction mapping principle. Also, he studied the global existence and decay results. Moreover, under suitable conditions, the author proved the blow-up results with $E(0)<0$.

In [17], Messaoudi studied the equation as follows:

$$
u_{t t}+\Delta^{2} u+\left|u_{t}\right|^{m-2} u_{t}=|u|^{p-2} u
$$

and obtained the existence results and obtained that, if $m \geq p$, the solution is global and blows up in finite time if $m<p$. Later, Chen and Zhou [18] extended this result. In the presence of the strong damping term $\left(-\Delta u_{t}\right)$, Pișkin and Polat [19] proved the global existence and decay of solutions for equation (7). For more results about plate problems, see [20-22]. follows:

In [7], Nicaise and Pignotti studied the equation as

$$
u_{t t}-\Delta u+a_{0} u_{t}(x, t)+a u_{t}(x, t-\tau)=0,
$$

where $a_{0}, a>0$. They proved that, under the condition $0 \leq a$ $\leq a_{0}$, the system is exponentially stable. The authors obtained a sequence of delays that shows the solution is unstable in the case $a \geq a_{0}$. In the absence of delay, some other authors $[23,24]$ looked into exponential stability for equation (8). In [9], Xu et al., by using the spectral analysis approach, established the same result similar to [7] for the one space dimension.

In [25], Nicaise et al. studied the wave equation in one space dimension in the presence of time-varying delay. In this article, the authors showed the exponential stability results with the condition

$$
a \leq \sqrt{1-d} a_{0}
$$

where $d$ is a constant and

$$
\tau^{\prime}(t) \leq d<1, \quad \forall t>0
$$

In [26], Kafini and Messaoudi studied wave equations with delay and logarithmic terms as follows:

$$
u_{t t}-\Delta u+\mu_{1} u_{t}(x, t)+\mu_{2} u_{t}(x, t-\tau)=|u|^{p-2} u \log |u|^{k}
$$

The authors proved the local existence and blow-up results for equation (11).

In [27], Park considered the equation with delay and logarithmic terms as follows:

$$
u_{t t}-\Delta u+\alpha u_{t}(t)+\beta u_{t}(x, t-\tau)=u \ln |u|^{\gamma}
$$

The author showed the local and global existence results for equation (12). Also, the author investigated the decay and nonexistence results for equation (12). In recent years, some other authors investigate hyperbolic-type equations with delay terms (see [28-33]).

In this work, we studied the local existence, global existence, nonexistence, and stability results of plate equation (1) with delay and logarithmic terms, motivated by the above works. There is no research, to our best knowledge, related to plate equation (1) with the delay $\left(\beta u_{t}(x, t-\tau)\right)$ term and logarithmic $\left(u \ln |u|^{\gamma}\right)$ source term; hence, our work is the generalization of the above studies.

This work consists of five sections in addition to the introduction. Firstly, in Section 2, we recall some assumptions and lemmas. Then, in Section 3, we obtain the local and global existence of solutions. Moreover, in Section 4, we establish the nonexistence results. Finally, in Section 5, we get the stability of solutions.

\section{Preliminaries}

In this part, we show the norm of $X$ by $\|\cdot\|_{X}$ for a Banach space $X$. We give the scalar product in $L^{2}(\Omega)$ by $(\cdot, \cdot)$. We show $\|\cdot\|_{2}$ by $\|\cdot\|$, for brevity. Let $B_{1}$ be the constant of the embedding inequality

$$
\|u\|^{2} \leq B_{1}\|\Delta u\|^{2} \quad \text { for } u \in H_{0}^{2}(\Omega) .
$$

We have the following assumptions related to problem (1):

(H1). The weights of delay and dissipation satisfy

$$
0<|\beta|<\alpha
$$

(H2). The constant $\gamma$ in (1) satisfies

$$
0<\gamma<\pi e^{(2(N+1)) / N}
$$

To get the main result, we have the lemmas as follows. 
Lemma 1 (see $[34,35])$ (Logarithmic Sobolev inequality). For any $u \in H_{0}^{1}(\Omega)$,

$\int_{\Omega} u^{2} \ln |u| d x \leq \frac{1}{2}\|u\|^{2} \ln \|u\|^{2}+\frac{k^{2}}{2 \pi}\|\nabla u\|^{2}-\frac{N}{2}(1+\ln k)\|u\|^{2}$

where $k$ is a positive real number.

Corollary 2. For any $u \in H_{0}^{2}(\Omega)$,

$\int_{\Omega} u^{2} \ln |u| d x \leq \frac{1}{2}\|u\|^{2} \ln \|u\|^{2}+\frac{k^{2}}{2 \pi}\|\Delta u\|_{2}^{2}-\frac{N}{2}(1+\ln k)\|u\|^{2}$,

where $k$ is a positive real number.

Remark 3. Assume that inequality (17) holds for all $k>0$, and we choose the constant $k$ that satisfies

$$
\rho=\max \left\{e^{-(N+1) / N}, \mu^{1 / N} \sqrt{\frac{\pi}{\gamma}}\right\}<k<\sqrt{\frac{\pi}{\gamma}},
$$

where $\mu$ is any real number with

$$
0<\mu<1
$$

Lemma 4 (see [12]) (Logarithmic Gronwall inequality). Suppose that $c>0$ and $l \in L^{1}\left(0, T ; R^{+}\right)$. If a function $f:[0, T]$ $\longrightarrow[1, \infty)$ satisfies

$$
f(t) \leq c\left(1+\int_{0}^{t} l(s) f(s) \ln f(s) d s\right), \quad 0 \leq t \leq T,
$$

then

$$
f(t) \leq c e^{c \int_{0}^{t} l(s) d s}, \quad 0 \leq t \leq T .
$$

We define

$$
\begin{aligned}
& J(v)=\frac{1}{2}\|\Delta v\|^{2}-\frac{1}{2} \int_{\Omega} v^{2} \ln |v|^{\gamma} d x+\frac{\gamma}{4}\|v\|^{2}, \\
& I(v)=\|\Delta v\|^{2}-\int_{\Omega} v^{2} \ln |v|^{\gamma} d x,
\end{aligned}
$$

for $v \in H_{0}^{2}(\Omega)$; then,

$$
J(v)=\frac{1}{2} I(v)+\frac{\gamma}{4}\|v\|^{2}
$$

Suppose that

$$
d=\inf _{v \in H_{0}^{2}(\Omega) \backslash\{0\}} \sup _{\lambda \geq 0} J(\lambda v) .
$$

Then, it satisfies (see, e.g., [36-38])

$$
0<d=\inf _{v \in \mathcal{N}} J(v)
$$

where $\mathcal{N}$ is the well-known Nehari manifold, denoted by

$$
\mathcal{N}=\left\{v \in H_{0}^{2}(\Omega) \backslash\{0\} \mid I(v)=0\right\} .
$$

Lemma 5. I and $J$ are the functions that satisfy

$$
I(\lambda v)=\lambda \frac{\partial J(\lambda v)}{\lambda v}\left\{\begin{array}{l}
>0,0<\lambda<\lambda^{*} \\
=0, \lambda=\lambda^{*} \\
<0, \lambda>\lambda^{*}
\end{array}\right.
$$

for any $v \in H_{0}^{2}(\Omega)$ with $\|v\| \neq 0$, where

$$
\lambda^{*}=\exp \left(\frac{\|\Delta v\|^{2}-\int_{\Omega} v^{2} \ln |v|^{\gamma} d x}{\gamma\|v\|^{2}}\right)
$$

Proof. We obtain, for $\lambda \geq 0$,

$$
\begin{aligned}
\lambda \frac{\partial}{\partial \lambda} J(\lambda v)= & \lambda\left\{\lambda\|\Delta v\|^{2}-\lambda \int_{\Omega} v^{2} \ln |v|^{\gamma} d x+\frac{\gamma \lambda}{2}\|v\|^{2}\right. \\
& \left.-\lambda \int_{\Omega} v^{2} \ln |\lambda|^{\gamma} d x-\frac{\gamma \lambda}{2} \int_{\Omega} v^{2} d x\right\} \\
= & \lambda^{2}\left(\|\Delta v\|^{2}-\int_{\Omega} v^{2} \ln |v|^{\gamma} d x-\gamma \ln |\lambda| \int_{\Omega} v^{2} d x\right) \\
= & I(\lambda v),
\end{aligned}
$$

and therefore, we obtain the desired result.

Remark 6. $J(\lambda v)$ has the absolute maximum value at $\lambda^{*}$, such that

$$
\sup _{\lambda \geq 0} J(\lambda v)=J\left(\lambda^{*} v\right)=\exp \left(\frac{2\|\Delta v\|^{2}-2 \int_{\Omega} v^{2} \ln |v|^{\gamma} d x}{\gamma\|v\|^{2}}\right) \frac{\gamma}{4}\|v\|^{2},
$$

for $v \in H_{0}^{2}(\Omega)$.

Lemma 7. The potential depth $d$ in (25) satisfies

$$
d \geq \frac{\gamma}{4} e^{N}\left(\frac{\pi}{\gamma}\right)^{N / 2}=E_{1}
$$


Proof. By Corollary 2, (13), and (18), we have

$$
\begin{aligned}
I(v) & \geq\left(1-\frac{k^{2} \gamma}{2 \pi}\right)\|\Delta v\|^{2}+\frac{N \gamma}{2}(1+\ln k)\|v\|^{2}-\frac{\gamma}{2}\|v\|^{2} \ln \|v\|^{2} \\
& >\frac{N \gamma}{2}(1+\ln k)\|v\|^{2}-\frac{\gamma}{2}\|v\|^{2} \ln \|v\|^{2} .
\end{aligned}
$$

Taking the limit $k \longrightarrow \sqrt{\pi / \gamma}$, we obtain

$$
I(v) \geq\left\{\frac{N \gamma}{2}\left(1+\ln \sqrt{\frac{\pi}{\gamma}}\right)-\frac{\gamma}{2} \ln \|v\|^{2}\right\}\|v\|^{2} .
$$

Taking into consideration this and (28), we get

$$
0=I\left(\lambda^{*} v\right) \geq\left\{\frac{N \gamma}{2}\left(1+\ln \sqrt{\frac{\pi}{\gamma}}\right)-\frac{\gamma}{2} \ln \left\|\lambda^{*} v\right\|^{2}\left\|\lambda^{*} v\right\|^{2}\right\},
$$

and therefore,

$$
\left\|\lambda^{*} v\right\|^{2} \geq e^{N}\left(\frac{\pi}{\gamma}\right)^{N / 2}
$$

Hence, we have by (24) and (31)

$\sup _{\lambda \geq 0} J(\lambda v)=J\left(\lambda^{*} v\right)=\frac{1}{2} I\left(\lambda^{*} v\right)+\frac{\gamma}{4}\left\|\lambda^{*} v\right\|^{2}=\frac{\gamma}{4}\left\|\lambda^{*} v\right\|^{2} \geq \frac{\gamma}{4} e^{N}\left(\frac{\pi}{\gamma}\right)^{N / 2}$.

From the definition of $d$ given in (25), we obtain the result.

\section{Existence}

In this part, we have studied the local existence, global existence, nonexistence, and stability results of plate equation (1) with delay and logarithmic terms, motivated by the above works. There is no research, to our best knowledge, related to plate equation (1) with the delay $\left(\beta u_{t}(x, t-\tau)\right)$ term and logarithmic $\left(u \ln |u|^{\gamma}\right)$ source term; hence, our work is the generalization of the above studies. Firstly, we introduce the new function

$$
y(x, \eta, t)=u_{t}(x, t-\eta \tau) \quad \text { for }(x, \eta, t) \in \Omega \times[0,1] \times(0, \infty) .
$$

Hence, problem (1) takes the form

$$
\begin{cases}u_{t t}+\Delta^{2} u+\alpha u_{t}(x, t)+\beta y(x, 1, t)=u \ln |u|^{\gamma} & \text { for }(x, t) \in \Omega \times(0, \infty), \\ \tau y_{t}(x, \eta, t)+y_{\eta}(x, \eta, t)=0 & \text { for }(x, \eta, t) \in \Omega \times(0,1) \times(0, \infty), \\ u(x, t)=\frac{\partial u(x, t)}{\partial v}=0 & \text { for }(x, t) \in \partial \Omega \times(0, \infty), \\ u(x, 0)=u_{0}(x), u_{t}(x, 0)=u_{1}(x) & \text { for } x \in \Omega, \\ y(x, \eta, 0)=j_{0}(x,-\eta \tau)=y_{0}(x, \eta) & \text { for }(x, \eta) \in \Omega \times(0,1) .\end{cases}
$$

Definition 8. Assume that $T>0 .(u, y)$ is a local solution of problem (39) if it satisfies

$$
\begin{gathered}
u \in C\left([0, T] ; H_{0}^{2}(\Omega)\right) \cap C^{1}\left([0, T] ; L^{2}(\Omega)\right) \cap C^{2}\left([0, T] ; H^{-2}(\Omega)\right), \\
\begin{aligned}
\left(u_{t t}, v\right)+(\Delta u, \Delta v)+\alpha\left(u_{t}(t), v\right)+\beta(y(1, t), v) \\
=\left(u \ln |u|^{\gamma}, v\right) \quad \text { for any } v \in H_{0}^{2}(\Omega), \\
\tau \int_{0}^{1}\left(y_{t}(\eta, t), \varphi(\eta)\right) d \eta+\int_{0}^{1}\left(y_{\eta}(\eta, t), \varphi(\eta)\right) d \eta \\
=0 \quad \text { for any } \varphi \in L^{2}(\Omega \times(0,1)), \\
u(0)=u_{0} \quad \text { in } H_{0}^{2}(\Omega), \\
u_{t}(0)=u_{1} \quad \text { in } L^{2}(\Omega), \\
y(0)=y_{0} \quad \text { in } L^{2}(\Omega \times(0,1)) .
\end{aligned}
\end{gathered}
$$

3.1. Local Existence. In this part, we establish the local existence results similar to $[8,39]$.

Theorem 9. Suppose that (H1) and (H2) are satisfied. Then, for the initial data $u_{0} \in H_{0}^{2}(\Omega), u_{1} \in L^{2}(\Omega)$, and $y_{0} \in L^{2}(\Omega \times$ $(0,1))$, there exists a local solution $(u, y)$ for problem (39).

Proof. Let $\left\{v_{i}\right\}_{i \in N}$ be the orthogonal basis of $H_{0}^{2}(\Omega)$ that is orthonormal in $L^{2}(\Omega)$. Define $\varphi_{i}(x, 0)=v_{i}(x)$, and we extend $\varphi_{i}(x, 0)$ by $\varphi_{i}(x, \eta)$ over $L^{2}(\Omega \times(0,1))$. We denote $V_{n}=$ $\operatorname{span}\left\{v_{1}, v_{2}, \cdots, v_{n}\right\}$ and $W_{n}=\operatorname{span}\left\{\varphi_{1}, \varphi_{2}, \cdots, \varphi_{n}\right\}$ for $n \geq 1$. We consider the Faedo-Galerkin approximation solution $\left(u^{n}, y^{n}\right) \in V_{n} \times W_{n}$ of the form

$$
\begin{aligned}
u^{n} & =\sum_{i=1}^{n} h_{i}^{n}(t) v_{i}(x), \\
y^{n}(x, \eta, t) & =\sum_{i=1}^{n} g_{i}^{n}(t) \varphi_{i}(x, \eta), \quad n=1,2, \cdots,
\end{aligned}
$$

solving the approximate system

$$
\begin{gathered}
\left(u_{t t}^{n}, v\right)+\left(\Delta u^{n}, \Delta v\right)+\alpha\left(u_{t}^{n}(t), v\right)+\beta\left(y^{n}(1, t), v\right) \\
=\int_{\Omega} u^{n} \ln \left|u^{n}\right|^{\gamma} v d x \quad \text { for } v \in V_{n},
\end{gathered}
$$




$$
\tau \int_{0}^{1}\left(y_{t}^{n}(\eta, t), \varphi(\eta)\right) d \eta+\int_{0}^{1}\left(y_{\eta}^{n}(\eta, t), \varphi(\eta)\right) d \eta=0 \quad \text { for } \varphi \in W_{n},
$$

$$
\begin{aligned}
& u^{n}(0)=u_{0}^{n}, \\
& u_{t}^{n}(0)=u_{1}^{n}, \\
& y^{n}(0)=y_{0}^{n},
\end{aligned}
$$

where

$$
\begin{array}{ll}
u_{0}^{n} \longrightarrow u_{0} & \text { in } H_{0}^{2}(\Omega), \\
u_{1}^{n} \longrightarrow u_{1} & \text { in } L^{2}(\Omega), \\
y_{0}^{n} \longrightarrow y_{0} & \text { in } L^{2}(\Omega \times(0,1)) .
\end{array}
$$

Since problem (42)-(44) is a normal system of ordinary differential equations, there exists a solution $\left(u^{n}, y^{n}\right)$ on the interval $\left[0, t_{n}\right), t_{n} \in(0, T]$. The extension of that solution to the $[0, T)$ is a consequence of the estimate below.

By replacing $v$ by $u_{t}^{n}(t)$ in (42) and by using the relation

$$
\int_{\Omega} u^{n} \ln \left|u^{n}\right|^{\gamma} u_{t}^{n} d x=\frac{d}{d t}\left\{\frac{1}{2} \int_{\Omega}\left(u^{n}\right)^{2} \ln \left|u^{n}\right|^{\gamma} d x-\frac{\gamma}{4}\left\|u^{n}\right\|^{2}\right\},
$$

we have

$$
\begin{aligned}
& \frac{d}{d t}\left\{\frac{1}{2}\left\|u_{t}^{n}\right\|^{2}+\frac{1}{2}\left\|\Delta u^{n}\right\|^{2}+\frac{\gamma}{4}\left\|u^{n}\right\|^{2}-\frac{1}{2} \int_{\Omega}\left(u^{n}\right)^{2} \ln \left|u^{n}\right|^{\gamma} d x\right\} \\
& \quad=-\alpha\left\|u_{t}^{n}(t)\right\|^{2}-\beta\left(y^{n}(1, t), u_{t}^{n}(t)\right) .
\end{aligned}
$$

By replacing $\varphi$ by $\omega y^{n}(\eta, t)$ in (43), we see that

$$
\frac{\omega \tau}{2} \frac{d}{d t} \int_{\Omega} \int_{0}^{1}\left(y^{n}(x, \eta, t)\right)^{2} d \eta d x=-\frac{\omega}{2}\left\|y^{n}(1, t)\right\|^{2}+\frac{\omega}{2}\left\|y^{n}(0, t)\right\|^{2} .
$$

Summing (47) and (48), we obtain

$\frac{d}{d t} E^{n}(t)=-\alpha\left\|u_{t}^{n}\right\|^{2}-\beta\left(y^{n}(1, t), u_{t}^{n}(t)\right)-\frac{\omega}{2}\left\|y^{n}(1, t)\right\|^{2}+\frac{\omega}{2}\left\|y^{n}(0, t)\right\|^{2}$,

where

$$
\begin{aligned}
E^{n}(t)= & \frac{1}{2}\left\|u_{t}^{n}\right\|^{2}+\frac{1}{2}\left\|\Delta u^{n}\right\|^{2}+\frac{\gamma}{4}\left\|u^{n}\right\|^{2}-\frac{1}{2} \int_{\Omega}\left(u^{n}\right)^{2} \ln \left|u^{n}\right|^{\gamma} d x \\
& +\frac{\omega \tau}{2}\left\|y^{n}\right\|_{L^{2}(\Omega \times(0,1))}^{2}
\end{aligned}
$$

where

$$
|\beta|<\omega<2 \alpha-|\beta| .
$$

Utilizing Young's inequality and the fact that $y^{n}(x, 0, t)$ $=u_{t}^{n}(x, t)$, we obtain

$$
\frac{d}{d t} E^{n}(t) \leq-\left(\alpha-\frac{|\beta|}{2}-\frac{\omega}{2}\right)\left\|u_{t}^{n}\right\|^{2}-\left(\frac{\omega}{2}-\frac{|\beta|}{2}\right)\left\|y^{n}(1, t)\right\|^{2} \leq 0,
$$

$$
E^{n}(t)+C_{1} \int_{0}^{t}\left\|u_{t}^{n}(s)\right\|^{2} d s+C_{2} \int_{0}^{t}\left\|y^{n}(1, s)\right\|^{2} d s \leq E^{n}(0),
$$

where

$$
\begin{aligned}
& C_{1}=\alpha-\frac{|\beta|}{2}-\frac{\omega}{2}>0, \\
& C_{2}=\frac{\omega}{2}-\frac{|\beta|}{2}>0 .
\end{aligned}
$$

Taking into consideration this and Corollary 2, we have

$$
\begin{aligned}
\left\|u_{t}^{n}\right\|^{2}+ & \left(1-\frac{\gamma k^{2}}{2 \pi}\right)\left\|\Delta u^{n}\right\|^{2}+\frac{\gamma}{2}(1+N(1+\ln k))\left\|u^{n}\right\|^{2} \\
+ & 2 C_{1} \int_{0}^{t}\left\|u_{t}^{n}(s)\right\|^{2} d s+2 C_{2} \int_{0}^{t}\left\|y^{n}(1, s)\right\|^{2} d s+\omega \tau\left\|y^{n}\right\|_{L^{2}(\Omega \times(0,1))}^{2} \\
& \leq 2 E^{n}(0)+\frac{\gamma}{2}\left\|u^{n}\right\|^{2} \ln \left\|u^{n}\right\|^{2} .
\end{aligned}
$$

By using (18), we obtain

$$
\begin{aligned}
1-\frac{\gamma k^{2}}{2 \pi} & >0, \\
\frac{\gamma}{2}(1+N(1+\ln k)) & >0,
\end{aligned}
$$

and therefore,

$$
\begin{aligned}
\left\|u_{t}^{n}\right\|^{2} & +\left\|\Delta u^{n}\right\|^{2}+\left\|u^{n}\right\|^{2}+\int_{0}^{t}\left\|u_{t}^{n}(s)\right\|^{2} d s+\int_{0}^{t}\left\|y^{n}(1, s)\right\|^{2} d s \\
& +\left\|y^{n}\right\|_{L^{2}(\Omega \times(0,1))}^{2} \leq c_{1}\left(1+\left\|u^{n}\right\|^{2} \ln \left\|u^{n}\right\|^{2}\right),
\end{aligned}
$$

where the sequel $c_{j}, j=1,2, \cdots$, shows a positive constant. Also, we know that

$$
u^{n}(x, t)=u^{n}(x, 0)+\int_{0}^{t} u_{t}^{n}(x, s) d s .
$$

Utilizing Cauchy-Schwarz's inequality and (57), we obtain 


$$
\begin{aligned}
\left\|u^{n}(t)\right\|^{2} & =2\left\|u^{n}(0)\right\|^{2}+2 T \int_{0}^{t}\left\|u_{t}^{n}(s)\right\|^{2} d s \\
& \leq 2\left\|u^{n}(0)\right\|^{2}+2 T \int_{0}^{t} c_{1}\left(1+\left\|u^{n}(s)\right\|^{2} \ln \left\|u^{n}(s)\right\|^{2}\right) d s \\
& \leq c_{2}\left(1+\int_{0}^{t}\left\|u^{n}(s)\right\|^{2} \ln \left\|u^{n}(s)\right\|^{2} d s\right) .
\end{aligned}
$$

From Lemma 4, we arrive at

$$
\left\|u^{n}(t)\right\|^{2} \leq c_{3} e^{c_{4} T}
$$

$f(s)=s \ln s$ is the function which is continuous on $(0, \infty)$ , $\lim _{s \longrightarrow 0^{+}} f(s)=0, \lim _{s \longrightarrow+\infty} f(s)=+\infty$, and $f$ decreases on $\left(0, e^{-1}\right)$ and increases on $\left(e^{-1},+\infty\right)$; hence, we get by (57) and (60)

$$
\begin{aligned}
\left\|u_{t}^{n}\right\|^{2} & +\left\|\Delta u^{n}\right\|^{2}+\left\|u^{n}\right\|^{2}+\int_{0}^{t}\left\|u_{t}^{n}(s)\right\|^{2} d s+\int_{0}^{t}\left\|y^{n}(1, s)\right\|^{2} d s \\
& +\left\|y^{n}\right\|_{L^{2}(\Omega \times(0,1))}^{2} \leq c_{5} .
\end{aligned}
$$

Hence, there exists a subsequence of $\left\{\left(u^{n}, y^{n}\right)\right\}$, which we still denote $\left\{\left(u^{n}, y^{n}\right)\right\}$, such that

$$
\begin{gathered}
u^{n} \longrightarrow u \quad \text { weakly star in } L^{\infty}\left(0, T ; H_{0}^{2}(\Omega)\right), \\
u_{t}^{n} \longrightarrow u_{t} \quad \text { weakly star in } L^{\infty}\left(0, T ; L^{2}(\Omega)\right), \\
y^{n} \longrightarrow y \quad \text { weakly star in } L^{\infty}\left(0, T ; L^{2}(\Omega \times(0,1))\right), \\
y^{n}(1) \longrightarrow y(1) \quad \text { weakly in } L^{2}\left(0, T ; L^{2}(\Omega)\right) .
\end{gathered}
$$

Utilizing the Aubin-Lions compactness theorem, we conclude that

$$
\begin{array}{ll}
u^{n} \longrightarrow u & \text { strongly in } L^{2}\left(0, T ; L^{2}(\Omega)\right), \\
u^{n} \longrightarrow u & \text { a.e.in } \Omega \times(0, T) .
\end{array}
$$

The function $s \longrightarrow s \ln |s|^{\gamma}$ is continuous on $R$; hence,

$$
u^{n} \ln \left|u^{n}\right|^{\gamma} \longrightarrow u \ln |u|^{\gamma} \quad \text { a.e.in } \Omega \times(0, T) .
$$

Let

$$
\begin{aligned}
& \Omega_{1}=\left\{x \in \Omega|| u^{n} \mid<1\right\}, \\
& \Omega_{2}=\left\{x \in \Omega|| u^{n} \mid \geq 1\right\} .
\end{aligned}
$$

Thus, we obtain

$$
\begin{aligned}
\int_{\Omega}\left(u^{n} \ln \left|u^{n}\right|^{\gamma}\right)^{2} d x & =\gamma^{2}\left\{\int_{\Omega_{1}}\left(u^{n} \ln \left|u^{n}\right|\right)^{2} d x+\int_{\Omega_{2}}\left(u^{n} \ln \left|u^{n}\right|\right)^{2} d x\right\} \\
& \leq \gamma^{2}\left\{e^{-2}\left|\Omega_{1}\right|+e^{-2}\left(\frac{2}{q-2}\right)^{2} \int_{\Omega_{2}}\left(u^{n}\right)^{q} d x\right\} \text { for any } q>2,
\end{aligned}
$$

where we used

$$
\begin{aligned}
& |s \ln s| \leq \frac{1}{e} \quad \text { for } 0<s<1, \\
& s^{-\kappa} \ln s \leq \frac{1}{e \kappa} \quad \text { for } s \geq 1 \text { and } \kappa>0 .
\end{aligned}
$$

By (57) and (66), we conclude that

$$
\int_{\Omega}\left(u^{n} \ln \left|u^{n}\right|^{\gamma}\right)^{2} d x \leq \gamma^{2}\left\{e^{-2}\left|\Omega_{1}\right|+e^{-2}\left(\frac{2}{q-2}\right)^{2} B_{2}^{q}\left\|\Delta u^{n}\right\|^{q}\right\} \leq c_{6},
$$

where $B_{2}$ is the Sobolev imbedding constant of

$$
H_{0}^{2}(\Omega) \subset L^{q}(\Omega) \quad \text { for } q>2 \text {, if } N=1,2,3,4 ; 2<q<\frac{2 N}{N-4} \text {, if } N \geq 5 .
$$

Therefore, we get from (68)

$u^{n} \ln \left|u^{n}\right|^{\gamma} \quad$ which is uniformly bounded in $L^{\infty}\left(0, T ; L^{2}(\Omega)\right)$.

From the Lebesgue bounded convergence theorem, (64), and (70), we arrive at

$$
u^{n} \ln \left|u^{n}\right|^{\gamma} \longrightarrow u \ln |u|^{\gamma} \quad \text { strongly in } L^{2}\left(0, T ; L^{2}(\Omega)\right) .
$$

We pass the limit $m \longrightarrow \infty$ in (42) and (43). The remainder of the proof is standard and similar to $[39,40]$.

3.2. Global Existence. In this part, we obtain the global existence results for problem (39). For this goal, we define the energy functional of problem (39):

$$
\begin{aligned}
E(t)= & \frac{1}{2}\left\|u_{t}\right\|^{2}+\frac{1}{2}\|\Delta u\|^{2}+\frac{\gamma}{4}\|u\|^{2}-\frac{1}{2} \int_{\Omega} u^{2} \ln |u|^{\gamma} d x \\
& +\frac{\omega \tau}{2}\|y\|_{L^{2}(\Omega \times(0,1))}^{2}
\end{aligned}
$$

where $\omega$ is the positive constant given in (51). We see that

$$
\begin{aligned}
E(t)= & \frac{1}{2}\left\|u_{t}\right\|^{2}+J(u(t))+\frac{\omega \tau}{2}\|y\|_{L^{2}(\Omega \times(0,1))}^{2}=\frac{1}{2}\left\|u_{t}\right\|^{2} \\
& +\frac{1}{2} I(u(t))+\frac{\gamma}{4}\|u\|^{2}+\frac{\omega \tau}{2}\|y\|_{L^{2}(\Omega \times(0,1))}^{2} .
\end{aligned}
$$


By the same arguments similar to (52), we infer that

$$
\frac{d}{d t} E(t) \leq-C_{1}\left\|u_{t}\right\|^{2}-C_{2}\|y(1, t)\|^{2} \leq 0
$$

where $C_{1}$ and $C_{2}$, given in (54), are positive constants.

Lemma 10. Suppose that (H1) and (H2) are satisfied. If $E(0$ )$<d$ and $I\left(u_{0}\right)>0$, then the solution $u$ of problem (1) satisfies

$$
I(u(t))>0 \quad \text { for } t \in[0, T)
$$

where $T$ is the maximal existence time of the solutions.

Proof. We know that $I\left(u_{0}\right)>0$ and $u$ is continuous on $[0, T)$; hence, we have

$$
I(u(t))>0 \text { for some interval }\left[0, t_{1}\right) \in[0, T) .
$$

Let $t_{0}$ be the maximum of $t_{1}$ satisfying (76). Assume that $t_{0}<T$; then, $I\left(u\left(t_{0}\right)\right)=0$, that is,

$$
u\left(t_{0}\right) \in \mathcal{N}
$$

Therefore, we obtain by (26)

$$
J\left(u\left(t_{0}\right)\right) \geq \inf _{v \in \mathscr{N}} J(v)=d .
$$

We see that this is in contradiction to the relation as follows:

$$
J\left(u\left(t_{0}\right)\right) \leq E\left(t_{0}\right) \leq E(0)<d .
$$

By (74) and Lemma 10 , we see that $E(t)$ is a nonincreasing function.

Theorem 11. The solution $u$ is global, under the conditions of Lemma 10.

Proof. It suffices to show that $\left\|u_{t}\right\|^{2}+\|\Delta u\|^{2}$ is bounded independent of $t$. By Lemma 10, (73), and (74), we get

$$
\left\|u_{t}\right\|^{2} \leq\left\|u_{t}\right\|^{2}+I(u(t)) \leq 2 E(t) \leq 2 E(0)<2 d .
$$

In a similar way, we get

$\|u\|^{2}<\|u\|^{2}+\frac{2}{\gamma} I(u(t))=\frac{4}{\gamma} J(u(t)) \leq \frac{4}{\gamma} E(t) \leq \frac{4}{\gamma} E(0)<\frac{4 d}{\gamma}$.

By Corollary 2 and (23), we conclude that

$$
\begin{aligned}
\|\Delta u\|^{2}= & I(u(t))+\gamma \int_{\Omega} u^{2} \ln |u| d x \leq 2 E(t)+\frac{\gamma}{2}\|u\|^{2} \ln \|u\|^{2} \\
& +\frac{k^{2} \gamma}{2 \pi}\|\Delta u\|^{2}-\frac{N \gamma}{2}(1+\ln k)\|u\|^{2}
\end{aligned}
$$

By taking the limit $k \longrightarrow \rho^{+}$in this inequality and from (81), we obtain

$$
\begin{aligned}
\left(1-\frac{\rho^{2} \gamma}{2 \pi}\right)\|\Delta u\|^{2} & \leq 2 E(t)+\frac{\gamma}{2}\left(\ln \|u\|^{2}-N(1+\ln \rho)\right)\|u\|^{2} \\
& <2 d+\frac{\gamma}{2}\left(\ln \left(\frac{4 d}{\gamma}\right)\right)-N(1+\ln \rho)\|u\|^{2} \\
& =2 d+\frac{\gamma}{2}\left\{\ln \left(\frac{4 d}{\gamma} e^{-N} \rho^{-N}\right)\right\}\|u\|^{2}
\end{aligned}
$$

By Lemma 7 and (18), we get

$$
\begin{aligned}
\ln \left(\frac{4 d}{\gamma} e^{-N} \rho^{-N}\right) & \geq \ln \left(\left(\frac{\pi}{\gamma}\right)^{N / 2} \rho^{-N}\right) \\
& =\ln \left(\left(\sqrt{\frac{\pi}{\gamma}} \rho^{-1}\right)^{N}\right) \ln 1=0 .
\end{aligned}
$$

Therefore, we see by (81) and (83) that

$$
\left(1-\frac{\rho^{2} \gamma}{2 \pi}\right)\|\Delta u\|^{2} \leq 2 d+2 d \ln \left(\frac{4 d}{\gamma} e^{-N} \rho^{-N}\right) .
$$

Hence, we conclude that

$$
\|\Delta u\|^{2}<2 d\left(1-\frac{\rho^{2} \gamma}{2 \pi}\right)^{-1}\left(1+\ln \left(\frac{4 d}{\gamma} e^{-N} \rho^{-N}\right)\right) .
$$

Therefore, we complete the proof by (80) and (86).

\section{Nonexistence}

In this part, similar to [41-43], we get the nonexistence results for problem (1). Firstly, we need the lemma as follows.

Lemma 12. Assume that (H1) and (H2) are satisfied. If $E(0)$ $<E_{1}$ and $I\left(u_{0}\right)<0$, then the solution $u$ of problem (1) satisfies

$$
I(u(t))<0 \quad \text { for } t \in[0, T),
$$

$$
\|u(t)\|^{2}>\frac{4 E_{1}}{\gamma} \quad \text { for } t \in[0, T)
$$

where $T$ is the maximal existence time of the solutions.

Proof. We know that $I\left(u_{0}\right)<0$ and $u$ is continuous on $[0, T)$; hence, we have

$$
I(u(t))<0 \quad \text { for some interval }\left[0, t_{1}\right) \subset[0, T)
$$

Let $t_{0}$ be the maximal time satisfying (89) and assume that $t_{0}<T$; then, $I\left(u_{0}\right)=0$, such that

$$
u\left(t_{0}\right) \in \mathcal{N}
$$


Therefore, we obtain

$d \leq J\left(u\left(t_{0}\right)\right)=\frac{1}{2} I\left(u\left(t_{0}\right)\right)+\frac{\gamma}{4}\left\|u\left(t_{0}\right)\right\|^{2} \leq E\left(u\left(t_{0}\right)\right) \leq E(0)<E_{1}$.

This is in contradiction to Lemma 7. Thus, (87) is proved. By Lemma 7, (31), and (87), we conclude that

$$
\begin{aligned}
E_{1} & \leq d \leq J\left(\lambda^{*} u(t)\right)=\exp \left(\frac{2\|\Delta u\|^{2}-2 \int_{\Omega} u^{2} \ln |u|^{\gamma} d x}{\gamma\|u\|^{2}}\right) \frac{\gamma}{4}\|u\|^{2} \\
& <\frac{\gamma}{4}\|u\|^{2} .
\end{aligned}
$$

Therefore, the proof is completed.

Theorem 13. Suppose that (H1) and (H2) are satisfied. Let $E(0)<\zeta E_{1}$, where $0<\zeta<1$, and $I\left(u_{0}\right)<0$. Then, the solution of problem (1) blows up at infinity.

Proof. Firstly, we set

$$
F(t)=\zeta E_{1}-E(t)
$$

By (74), we obtain

$$
F^{\prime}(t)=-E^{\prime}(t) \geq C_{1}\left\|u_{t}\right\|^{2}+C_{2}\|y(1, t)\|^{2} \geq 0 .
$$

Utilizing (72), (88), and (94), we see that

$$
\begin{aligned}
0< & F(0) \leq F(t) \leq \zeta E_{1}+\frac{1}{2} \int_{\Omega} u^{2} \ln |u|^{\gamma} d x<\frac{\gamma}{4}\|u\|^{2} \\
& +\frac{1}{2} \int_{\Omega} u^{2} \ln |u|^{\gamma} d x .
\end{aligned}
$$

We define

$$
G(t)=F(t)+\varepsilon\left(u, u_{t}\right)+\frac{\varepsilon \alpha}{2}\|u\|^{2}
$$

By (39) and (72), we get

$$
\begin{aligned}
G^{\prime}(t)= & F^{\prime}(t)+\varepsilon\left\|u_{t}\right\|^{2}-\varepsilon\|\Delta u\|^{2}-\varepsilon \beta(u, y(1, t)) \\
& +\varepsilon \int_{\Omega} u^{2} \ln |u|^{\gamma} d x=F^{\prime}(t)+2 \varepsilon\left\|u_{t}\right\|^{2}-\varepsilon \beta(u, y(1, t)) \\
& -2 \varepsilon E(t)+\frac{\varepsilon \gamma}{2}\|u\|^{2}+\omega \tau\|y\|_{L^{2}(\Omega \times(0,1))}^{2}
\end{aligned}
$$

Utilizing Young's inequality and (94), we obtain

$$
\beta(u, y(1, t)) \leq|\beta|\left(\delta\|u\|^{2}+\frac{1}{4 \delta}\|y(1, t)\|^{2}\right) \leq \delta|\beta|\|u\|^{2}+\frac{|\beta|}{4 \delta C_{2}} F^{\prime}(t) .
$$

By adapting this to (97) and from (88) and (93), we have

$$
\begin{aligned}
G^{\prime}(t) \geq & \left(1-\frac{\varepsilon|\beta|}{4 \delta C_{2}}\right) F^{\prime}(t)+2 \varepsilon\left\|u_{t}\right\|^{2}+\left(\frac{\varepsilon \gamma}{2}-\varepsilon|\beta| \delta\right)\|u\|^{2} \\
& +2 \varepsilon F(t)-2 \varepsilon \zeta E_{1}+\omega \tau\|y\|_{L^{2}(\Omega \times(0,1))}^{2} \\
\geq & \left(1-\frac{\varepsilon|\beta|}{4 \delta C_{2}}\right) F^{\prime}(t)+2 \varepsilon\left\|u_{t}\right\|^{2}+\varepsilon\left((1-\zeta) \frac{\gamma}{2}-|\beta| \delta\right)\|u\|^{2} \\
& +2 \varepsilon F(t)+\omega \tau\|y\|_{L^{2}(\Omega \times(0,1))}^{2} .
\end{aligned}
$$

Firstly, fix $\delta>0$ such that $(1-\zeta)(\gamma / 2)-|\beta| \delta>0$ and then choose $\varepsilon>0$ small enough so that $1-\left(\varepsilon|\beta| / 4 \delta C_{2}\right)>0$. Then, by (94), we get

$$
G^{\prime}(t) \geq c_{8}\left(F(t)+\left\|u_{t}\right\|^{2}+\|u\|^{2}\right) \geq 0 .
$$

Also, we conclude that

$$
G(t) \leq c_{9}\left(F(t)+\left\|u_{t}\right\|^{2}+\|u\|^{2}\right) .
$$

Taking $\varepsilon>0$ small enough again, we obtain

$$
G(0)=F(0)+\varepsilon\left(u_{0}, u_{1}\right)+\frac{\varepsilon \alpha}{2}\left\|u_{0}\right\|^{2}>0 .
$$

By (100) and (102), we get

$$
G(t) \geq G(0)>0 \text {. }
$$

Utilizing (100) and (101), we see that

$$
G^{\prime}(t) \geq c_{10} G(t)
$$

and therefore,

$$
G(t) \geq e^{c_{10} t} G(0)>0 .
$$

Therefore, $G(t)$ blows up at infinity. Consequently, the proof is completed.

\section{Stability}

In this part, we obtain the stability of global solutions. Firstly, we define the perturbed energy by

$$
\Psi(t)=E(t)+\varepsilon \Phi(t)+\varepsilon \Xi(\mathrm{t})
$$

where $\varepsilon>0, \Phi(t)=\left(u_{t}, u\right)$, and $\Xi(t)=\int_{\Omega} \int_{0}^{1} e^{-\tau \eta} y^{2}(x, \eta, t) d \eta$ $d x$.

Lemma 14. Under the conditions of Lemma 10, for $C_{3}, C_{4}>0$, we obtain

$$
C_{3} E(t) \leq \Psi(t) \leq C_{4} E(t)
$$


Proof. Utilizing Lemma 10 and Young's inequality, we have

$$
\begin{aligned}
|\Phi(t)+\Xi(t)| \leq & \frac{1}{2}\left\|u_{t}\right\|^{2}+\frac{1}{2}\|u\|^{2}+\|y\|_{L^{2}(\Omega \times(0,1))}^{2} \\
\leq & \frac{1}{2}\left\|u_{t}\right\|^{2}+\frac{2}{\gamma}\left(\frac{\gamma}{4}\|u\|^{2}+\frac{1}{2} I(u(t))\right) \\
& +\|y\|_{L^{2}(\Omega \times(0,1))}^{2}=\frac{1}{2}\left\|u_{t}\right\|^{2}+\frac{2}{\gamma} J(u(t)) \\
& +\|y\|_{L^{2}(\Omega \times(0,1))}^{2} \leq c_{7} E(t) .
\end{aligned}
$$

Taking $\varepsilon>0$ small enough, we complete the proof.

Theorem 15. Assume that (H1) and (H2) are satisfied. Suppose that $E(0)<E_{1}$ and $I\left(u_{0}\right)>0$. Hence, for $C_{0}, C_{5}>0$, we obtain

$$
0<E(t) \leq C_{0} e^{-C_{5} t} \quad \text { for } t \geq 0 .
$$

Proof. From (39) and Young's inequality, we get

$$
\begin{aligned}
\Phi^{\prime}(t)= & \left\|u_{t}\right\|^{2}-\|\Delta u\|^{2}-\alpha\left(u_{t}(t), u(t)\right)-\beta(y(1, t), u(t)) \\
& +\int_{\Omega} u^{2} \ln |u|^{\gamma} d x \leq\left\|u_{t}\right\|^{2}-\frac{1}{2}\|\Delta u\|^{2}+\alpha^{2} B_{1}\left\|u_{t}(t)\right\|^{2} \\
& +\beta^{2} B_{1}\|y(1, t)\|^{2}+\int_{\Omega} u^{2} \ln |u|^{\gamma} d x .
\end{aligned}
$$

By using the second equation of (39) and the integration by parts, we obtain

$$
\begin{aligned}
\Xi^{\prime}(t) & =-\frac{2}{\tau} \int_{\Omega} \int_{0}^{1} e^{-\tau \eta} y(x, \eta, t) y_{\eta}(x, \eta, t) d \eta d x \\
& =-\frac{1}{\tau} \int_{\Omega} \int_{0}^{1} e^{-\tau \eta} \frac{\partial}{\partial \eta} y^{2}(x, \eta, t) d \eta d x \\
& =-\frac{e^{-\tau}}{\tau}\|y(1, t)\|^{2}+\frac{1}{\tau}\|y(0, t)\|^{2}-\int_{\Omega} \int_{0}^{1} e^{-\tau \eta} y^{2}(x, \eta, t) d \eta d x \\
& \leq \frac{1}{\tau}\left\|u_{t}\right\|^{2}-e^{-\tau} \int_{\Omega} \int_{0}^{1} y^{2}(x, \eta, t) d \eta d x .
\end{aligned}
$$

Summing these and (74), we obtain

$$
\begin{aligned}
\Psi^{\prime}(t) \leq & -\left(C_{1}-\varepsilon-\varepsilon \alpha^{2} B_{1}-\frac{\varepsilon}{\tau}\right)\left\|u_{t}\right\|^{2}-\frac{\varepsilon}{2}\|\Delta u\|^{2} \\
& -\left(C_{2}-\varepsilon \beta^{2} B_{1}\right)\|y(1, t)\|^{2}+\varepsilon \int_{\Omega} u^{2} \ln |u|^{\gamma} d x \\
& -\varepsilon e^{-\tau}\|y\|_{L^{2}(\Omega \times(0,1))}^{2}
\end{aligned}
$$

Adding and subtracting $\xi E(t)$ with $0<\xi<2 \varepsilon$, we get

$$
\begin{aligned}
\Psi^{\prime}(t) \leq & -\xi E(t)-\left(C_{1}-\varepsilon-\varepsilon \alpha^{2} B_{1}-\frac{\varepsilon}{\tau}-\frac{\xi}{2}\right)\left\|u_{t}\right\|^{2} \\
& -\left(\frac{\varepsilon}{2}-\frac{\xi}{2}-\frac{\xi \gamma B_{1}}{4}\right)\|\Delta u\|^{2}-\left(C_{2}-\varepsilon \beta^{2} B_{1}\right)\|y(1, t)\|^{2} \\
& +\left(\varepsilon-\frac{\xi}{2}\right) \int_{\Omega} u^{2} \ln |u|^{\gamma} d x-\left(\varepsilon e^{-\tau}-\frac{\xi \omega \tau}{2}\right)\|y\|_{L^{2}(\Omega \times(0,1))}^{2}
\end{aligned}
$$

Utilizing the logarithmic Sobolev inequality, we have

$$
\begin{aligned}
\Psi^{\prime}(t) \leq & -\xi E(t)-\left(C_{1}-\varepsilon-\varepsilon \alpha^{2} B_{1}-\frac{\varepsilon}{\tau}-\frac{\xi}{2}\right)\left\|u_{t}\right\|^{2} \\
& -\left\{\varepsilon\left(\frac{1}{2}-\frac{\gamma k^{2}}{2 \pi}\right)-\frac{\xi}{2}\left(1-\frac{\gamma k^{2}}{2 \pi}\right)-\frac{\xi \gamma B_{1}}{4}\right\}\|\Delta u\|^{2} \\
& +\frac{\gamma}{2}\left(\varepsilon-\frac{\xi}{2}\right)\left\{\ln \|u\|^{2}-N(1+\ln k)\right\}\|u\|^{2} \\
& -\left(C_{2}-\varepsilon \beta^{2} B_{1}\right)\|y(1, t)\|^{2}-\left(\varepsilon e^{-\tau}-\frac{\xi \omega \tau}{2}\right)\|y\|_{L^{2}(\Omega \times(0,1))}^{2}
\end{aligned}
$$

Now, choose $\varepsilon>0$ small enough, such that

$$
\begin{aligned}
C_{1}-\varepsilon-\varepsilon \alpha^{2} B_{1}-\frac{\varepsilon}{\tau}>0, \\
C_{2}-\varepsilon \beta^{2} B_{1}>0 .
\end{aligned}
$$

By taking $\xi>0$ sufficiently small and noting that $(1 / 2)$ $-\left(\gamma k^{2} / 2 \pi\right)>0$ (see (18)), we infer that

$$
\Psi^{\prime}(t) \leq-\xi E(t)+\frac{\gamma}{2}\left(\varepsilon-\frac{\xi}{2}\right)\left\{\ln \|u\|^{2}-N(1+\ln k)\right\}\|u\|^{2},
$$

where $0<E(0)<E_{1}$; therefore, there exists $0<\mu<1$, that is, $E(0)=\mu E_{1}$. Therefore, we obtain by $(81)$

$$
\begin{aligned}
\ln \|u\|^{2} & <\ln \left(\frac{4}{\gamma} E(t)\right) \leq \ln \left(\frac{4}{\gamma} E(0)\right)=\ln \left(\frac{4 \mu E_{1}}{\gamma}\right) \\
& =\ln \left(\mu e^{N}\left(\frac{\pi}{\gamma}\right)^{N / 2}\right) .
\end{aligned}
$$

Hence, by (18), we arrive at

$$
\begin{aligned}
\ln \|u\|^{2}-N(1+\ln k) & \leq \ln \left(\mu e^{N}\left(\frac{\pi}{\gamma}\right)^{N / 2}\right)-N(1+\ln k) \\
& =N \ln \left(\mu^{1 / N} \sqrt{\frac{\pi}{\gamma}} k^{-1}\right)<N \ln 1=0 .
\end{aligned}
$$


Substituting this into (116), we arrive at

$$
\Psi^{\prime}(t) \leq-\xi E(t)
$$

As a result, from Lemma 14, we completed the proof.

\section{Conclusions}

Recently, there have been many published works related to wave equations with time delay. There were no local existence, global existence, nonexistence, and stability results of the plate equation with delay and logarithmic source terms, to the best of our knowledge. Firstly, we have obtained the local and global existence results. Then, we have obtained the nonexistence of solutions. Finally, we have proved stability results under sufficient conditions.

\section{Data Availability}

No data were used to support the study.

\section{Conflicts of Interest}

The authors declare that they have no competing interests.

\section{Acknowledgments}

The first and second authors are grateful to DUBAP (ZGEF.20.009) for research funds.

\section{References}

[1] K. Bartkowski and P. Gŏrka, "One-dimensional Klein-Gordon equation with logarithmic nonlinearities," Journal of Physics A: Mathematical and Theoretical, vol. 41, no. 35, article 355201, 2008.

[2] P. Gorka, "Logarithmic Klein-Gordon equation," Acta Physica Polonica, vol. B40, pp. 59-66, 2009.

[3] C. Abdallah, P. Dorato, J. Benitez-Read, and R. Byrne, "Delayed positive feedback can stabilize oscillatory system," A.C.C. San Francisco, 1993.

[4] I. H. Suh and Z. Bien, "Use of time-delay actions in the controller design," IEEE Transactions on Automatic Control, vol. 25, no. 3, pp. 600-603, 1980.

[5] R. Datko, J. Lagnese, and M. P. Polis, "An example on the effect of time delays in boundary feedback stabilization of wave equations," SIAM Journal on Control and Optimization, vol. 24, no. 1, pp. 152-156, 1986.

[6] M. Kafini and S. A. Messaoudi, "A blow-up result in a nonlinear wave equation with delay," Mediterranean Journal of Mathematics, vol. 13, no. 1, pp. 237-247, 2016.

[7] S. Nicaise and C. Pignotti, "Stability and instability results of the wave equation with a delay term in the boundary or internal feedbacks," SIAM Journal on Control and Optimization, vol. 45, no. 5, pp. 1561-1585, 2006.

[8] S. Nicaise and C. Pignotti, "Stabilization of the wave equation with boundary or internal distributed delay," Differential and Integral Equations, vol. 21, pp. 935-958, 2008.

[9] C. Q. Xu, S. P. Yung, and L. K. Li, "Stabilization of the wave system with input delay in the boundary control," ESAIM, vol. 12, pp. 770-785, 2006.
[10] I. Bialynicki-Birula and J. Mycielski, "Wave equations with logarithmic nonlinearities," Bulletin de l'Academie Polonaise des Sciences. Serie des Sciences, Mathematiques, Astronomiques et Physiques, vol. 23, no. 4, pp. 461-466, 1975.

[11] I. Bialynicki-Birula and J. Mycielski, "Nonlinear wave mechanics," Annals of Physics, vol. 100, no. 1-2, pp. 62-93, 1976.

[12] T. Cazenave and A. Haraux, "Équations d'évolution avec non linéarité logarithmique," Annales de la Faculté des Sciences de Toulouse Mathématiques, vol. 2, no. 1, pp. 21-51, 1980.

[13] T. Hiramatsu, M. Kawasaki, and F. Takahashi, "Numerical study of q-ball formation in gravity mediation," Journal of Cosmology and Astroparticle Physics, vol. 2010, no. 6, p. 8, 2010.

[14] X. Han, "Global existence of weak solutions for a logarithmic wave equation arising from q-ball dynamics," Bulletin of the Korean Mathematical Society, vol. 50, no. 1, pp. 275-283, 2013.

[15] M. M. Al-Gharabli and S. A. Messaoudi, "Existence and a general decay result for a plate equation with nonlinear damping and a logarithmic source term," Journal of Evolution Equations, vol. 18, no. 1, pp. 105-125, 2018.

[16] G. Liu, “The existence, general decay and blow-up for a plate equation with nonlinear damping and a logarithmic source term," Electronic Research Archive, vol. 28, no. 1, pp. 263289, 2020.

[17] S. A. Messaoudi, "Global existence and nonexistence in a system of Petrovsky," Journal of Mathematical Analysis and Applications, vol. 265, no. 2, pp. 296-308, 2002.

[18] W. Chen and Y. Zhou, "Global nonexistence for a semilinear Petrovsky equation," Nonlinear Analysis: Theory, Methods \& Applications, vol. 70, no. 9, pp. 3203-3208, 2009.

[19] E. Pișkin and N. Polat, "On the decay of solutions for a nonlinear Petrovsky equation," Mathematical Sciences Letters, vol. 3, no. 1, pp. 43-47, 2013.

[20] V. Komornik, "On the nonlinear boundary stabilization of Kirchhoff plates," Nonlinear Differential Equations and Applications NoDEA, vol. 1, no. 4, pp. 323-337, 1994.

[21] J. Lagnese, "Asymptotic energy estimates for Kirchhoff plates subject to weak viscoelastic damping," in International Series of Numerical Mathematics, vol. 91, Birhauser, Verlag, Bassel, 1989.

[22] J. Muñoz Rivera, E. C. Lapa, and R. Barreto, "Decay rates for viscoelastic plates with memory," Journal of Elasticity, vol. 44, no. 1, pp. 61-87, 1996.

[23] K. Liu, "Locally distributed control and damping for the conservative systems," SIAM Journal on Control and Optimization, vol. 35, no. 5, pp. 1574-1590, 1997.

[24] E. Zuazua, "Exponential decay for the semilinear wave equation with locally distributed damping," Communications in Partial Differential Equations, vol. 15, pp. 205-235, 1990.

[25] S. Nicaise, J. Valein, and E. Fridman, "Stability of the heat and of the wave equations with boundary time-varying delays," Discrete \& Continuous Dynamical Systems - S, vol. 2, no. 3, pp. 559-581, 2009.

[26] M. Kafini and S. A. Messaoudi, "Local existence and blow up of solutions to a logarithmic nonlinear wave equation with delay," Applicable Analysis, vol. 99, pp. 530-547, 2019.

[27] S. H. Park, "Global existence, energy decay and blow-up of solutions for wave equations with time delay and logarithmic source," Advances in Difference Equations, vol. 2020, 17 pages, 2020. 
[28] E. Pișkin and H. Yüksekkaya, "Nonexistence of solutions of a delayed wave equation with variable-exponents," C-POST, vol. 3, no. 1, pp. 97-101, 2020.

[29] E. Pișkin and H. Yüksekkaya, "Decay and blow up of solutions for a delayed wave equation with variable-exponents," Conference Proceedings of Science and Technology, vol. 3, no. 1, pp. 91-96, 2020.

[30] E. Pișkin and H. Yüksekkaya, "Local existence and blow up of solutions for a logarithmic nonlinear viscoelastic wave equation with delay," Computational Methods for Differential Equations, vol. 9, no. 2, pp. 623-636, 2021.

[31] E. Piskin and H. Yüksekkaya, "Nonexistence of global solutions of a delayed wave equation with variable-exponents," Miskolc Math. Notes, vol. 22, pp. 1-19, 2021.

[32] E. Pișkin and H. Yüksekkaya, "Decay of solutions for a nonlinear Petrovsky equation with delay term and variable exponents," The Aligarh Bulletin Of Mathematics, vol. 39, no. 2, pp. 63-78, 2020.

[33] E. Pișkin and H. Yüksekkaya, "Blow-up of solutions for a logarithmic quasilinear hyperbolic equation with delay term," Journal of Mathematical Analysis and Applications, vol. 12, no. 1, pp. 56-64, 2021.

[34] H. Chen, P. Luo, and G. W. Liu, "Global solution and blow-up of a semilinear heat equation with logarithmic nonlinearity," Journal of Mathematical Analysis and Applications, vol. 422, no. 1, pp. 84-98, 2015.

[35] L. Gross, "Logarithmic Sobolev inequalities," American Journal of Mathematics, vol. 97, no. 4, pp. 1061-1083, 1975.

[36] H. Chen and G. Liu, "Global existence and nonexistence for semilinear parabolic equations with conical degeneration," Journal of Pseudo-Differential Operators and Applications, vol. 3, no. 3, pp. 329-349, 2012.

[37] Y. Liu, "On potential wells and applications to semilinear hyperbolic equations and parabolic equations," Nonlinear Analysis, vol. 64, pp. 2665-2687, 2006.

[38] L. Payne and D. Sattinger, "Saddle points and instability of nonlinear hyperbolic equation," Israel Journal of Mathematics, vol. 226, pp. 273-303, 1975.

[39] M. M. Al-Gharabli and S. A. Messaoudi, "The existence and the asymptotic behavior of a plate equation with frictional damping and a logarithmic source term," Journal of Mathematical Analysis and Applications, vol. 454, no. 2, pp. 11141128, 2017.

[40] J. L. Lions, Quelques Méthodes de Résolution des Problémes aux Limites Non Linéaires, Dunod, Paris, 1969.

[41] W. J. Liu and J. Yu, "On decay and blow-up of the solution for a viscoelastic wave equation with boundary damping and source terms," Nonlinear Analysis, vol. 74, no. 6, pp. 21752190, 2011.

[42] S. A. Messaoudi, "Blow up and global existence in a nonlinear viscoelastic wave equation," Mathematische Nachrichten, vol. 260, no. 1, pp. 58-66, 2003.

[43] S. H. Park, M. J. Lee, and J. R. Kang, "Blow-up results for viscoelastic wave equations with weak damping," Applied Mathematics Letters, vol. 80, pp. 20-26, 2018. 